\title{
Access granted: iRGD helps silicasome-encased drugs breach the tumor barrier
}

\author{
Erkki Ruoslahti
}

Cancer Research Center, Sanford Burnham Prebys Medical Discovery Institute, La Jolla, California, USA. Center for Nanomedicine and Department of Molecular Cellular and Developmental Biology, University of California, Santa Barbara, Santa Barbara, California, USA.

\begin{abstract}
In this issue of the $J C I$, Liu et al. use irinotecan-loaded nanoparticles to treat pancreatic adenocarcinomas in mice. Encapsulating drugs into nanoparticles has distinct advantages: it can improve the pharmacokinetics of the drug, enhance efficacy, and reduce unwanted side effects. A drawback is that the large size of nanoparticles restricts their access to the tumor interior. Liu and colleagues show that the cyclic tumor-penetrating peptide iRCD, reported to be capable of enhancing tumor penetration by drugs, can overcome this limitation to a substantial degree when administered together with the nanoparticles. Pancreatic adenocarcinoma is a challenging malignancy to treat and in desperate need for improved treatments; therefore, advances like this are most welcome.
\end{abstract}

\section{Improving drug delivery to tumor sites}

Pancreatic ductal adenocarcinoma (PDAC) is a particularly difficult to treat cancer with a dismal prognosis. A major factor in the lethality of PDAC is thought to be limited access of drugs into these tumors. PDAC tumors are highly fibrotic, consisting mostly of collagenous connective tissue (1). This tightly woven matrix forms a physical barrier for drug entry. In addition, high interstitial fluid pressure within tumors causes fluid (and drug) outflow from the tumor $(2,3)$. It is not surprising then that a significant effort is underway to facilitate drug entry into PDAC tumors (1). The entry barrier is a particularly acute problem with nanoparticle formulations because of their large size, but nanoparticle drugs often have lower toxicities than the corresponding free drugs and also tend to be more efficacious (4).

In this issue, Liu et al. tackle the above issues using tumor-targeted, drug-loaded nanoparticles in a number of mouse PDAC models (5). The nanoparticles tested were about $100 \mathrm{~nm}$ in diameter and composed of mesoporous silica, with the silica particle encased in a lipid bilayer. The entire structure has been dubbed a silicasome. This group reported earlier that silicasomes can be loaded with more drug than liposomes and that silicasome delivery of irinotecan and a paclitaxel-gemcitabine combination provides improved pharmacokinetics, greater treatment efficacy, and reduced toxicity compared with the corresponding liposomal drug in an orthotopic PDAC model $(6,7)$. Liu and colleagues also set out to deal with the issue of nanoparticle penetration by making use of peptide-mediated transcytosis (5).

The peptide Liu et al. employed is a tumor-penetrating peptide described as being capable of enhancing the accumulation and penetration of various drugs into tumors $(8,9)$. The peptide, iRGD, is a cyclic 9-amino acid peptide with a sequence of CRGDKGPDC, where the lysine can be an arginine and the aspar-

Related Article: p. 2007

Conflict of interest: E. Ruoslahti is an officer and shareholder of DrugCendR Inc., a company that holds a license for the iRCD peptide and is developing the peptide for clinical use. E. Ruoslahti is an inventor of patents covering the iRCD peptide. Reference information: J Clin Invest. 2017;127(5):1622-1624. https://doi.org/10.1172/JCI93955.

tic acid a glutamic acid and the cyclizing bond is between the cysteine side chains. The peptide contains two sequence motifs critical to its function: an integrin-binding RGD motif (10) and a neuropilin-1-binding (NRP-1-binding) C-terminal rule (CendR) motif $(\mathrm{R} / \mathrm{KXXR} / \mathrm{K})$, where $\mathrm{X}$ is any amino acid (11). The RGD motif mediates the binding of iRGD to $\alpha_{v}$ integrins, and as these integrins are specifically expressed on tumor blood vessels (and vessels undergoing angiogenesis in general), the RGD motif targets the iRGD peptide specifically to tumors. The CendR motif in iRGD is cryptic and only active at the $\mathrm{C}$ terminus of the peptide. Cend $\mathrm{R}$ is activated by a proteolytic cleavage at the surface of tumor endothelial cells that makes the RGDK/R sequence $\mathrm{C}$-terminal and thereby renders the peptide capable of binding to NRP-1. The binding of a peptide or a protein (certain forms of vascular endothelial growth factor and some semaphorins are such proteins) to NRP-1 through a C-terminal CendR motif activates an endocytic transcytosis and trans-tissue transport pathway. This pathway mediates uptake into cells, extravasation, and tumor penetration of the truncated peptide, which is also capable of taking a drug with it (Figure 1). At the individual cell level, iRGD induces NRP-1-dependent internalization of the peptide and accompanying drugs (thus the term internalizing RGD or iRGD) (8).

The uptake of CendR peptides is an endocytic process that resembles, but is distinct from, macropinocytosis (12). Interestingly, the activity of the pathway is increased by nutrient deprivation of cells and tissues, suggesting that the physiological role of this pathway may be to support the survival of tissues at times of poor nutrient availability. Cell-to-cell transport of the CendR payload has also been demonstrated (12). The payload can be chemically conjugated to iRGD. However, 


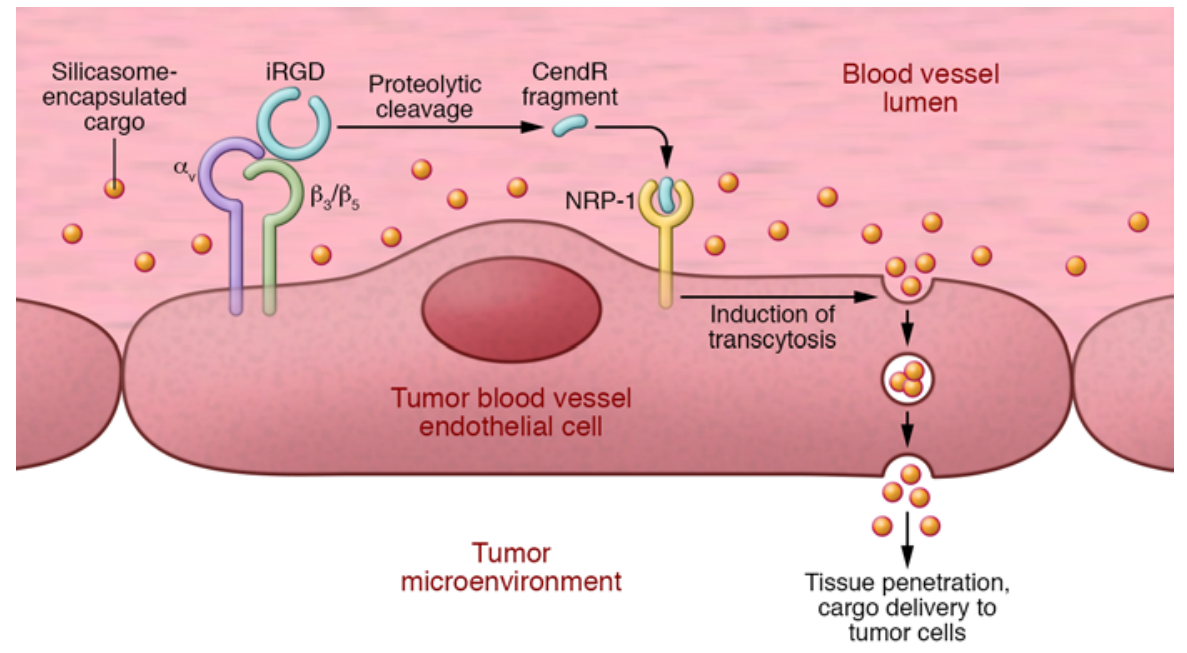

that does not have to be the case because the CendR pathway is a bulk transport system; therefore, any compound present in the blood can be swept into the transcytosis pathway (bystander effect).

\section{Harnessing the bystander effect for nanoparticle delivery}

A number of laboratories have made use of the iRGD bystander effect in targeting of a variety of anticancer therapeutics, including various chemotherapeutic agents, kinase inhibitors, antibodies, and even nanoparticles (refs. 9, 13-16; see ref. 17 for additional references). Liu et al. (5) tested both the iRGD-coupled and iRGD-coadministration approaches for effective delivery of silicasomes. In carefully conducted studies, Liu and colleagues found that, compared with administration of plain silicasomes or iRGD-conjugated silicasomes, iRGD coadministration enhanced tumor accumulation of silicasomes by a factor of three. Moreover, iRGD did not promote accumulation of these nanoparticles in normal tissues. The authors observed a bystander effect of similar magnitude in both an orthotopically transplanted PDAC model and a patient-derived xenograft (PDX) model. Remarkably, Liu et al. were able to visualize the iRGD-induced endocytic transport vesicles in the tumors by electron microscopy. The approximately 3 -fold difference in uptake as a result of iRGD coadministration is similar to what other laboratories have observed (17), although the difference in acute accumulation of nanoparticles can be much higher (9). Regarding the superior effect of iRGD coadministration, Sugahara et al. (9) found a similar trend in uptake of the nanoparticle drug nabpaclitaxel, but the difference between accumulation the conjugated and coadministered nabpaclitaxel particles was not statistically significant. A possible reason for the difference is that the relatively stable silicasomes may get caught in a so-called "binding site barrier" (18) because of the high affinity provided by the multivalent presentation of iRGD on their surface. In contrast, nabpaclitaxel disassembles in vivo, thereby losing the peptide multivalency. Of note, silicasomes do degrade slowly in the body, forming silicic acid, which is a nontoxic compound normally present in the body (19).

Results showing that iRGD coadministration enhances nanoparticle uptake at tumor sites were recently challenged by a reproducibility program study, which found no difference in doxorubicin tumor accumulation, whether doxorubicin was given with or without iRGD (20). It is difficult to reconcile the result of this very limited study with the dozen or so papers previously published by laboratories other than the originators of the system and with the latest study by Liu et al. in this issue (5). Among possible nontrivial reasons for the discrepancy, the most solid lead is the influence of the nutrient status of the target tumor on the CendR pathway (12). What the mice eat and what time of the day the injections are made could affect the results. This aspect of iRGD-enhanced therapy definitely merits further study.
Figure 1. iRGD-enhanced tumor accumulation of silicasome-encapsulated drugs. Intravenous iRGD penetrates tumor tissue in a 3-step process. First, iRGD binds to $\alpha_{v}$ integrins. Second, protease cleavage of bound iRGD generates a C-terminal CendR-containing fragment of iRCD at the tumor site. Third, CendR fragment binds to NRP-1, which promotes silicasome uptake and transcytosis through the vessel endothelium, allowing delivery of cargo directly to the target tumor.

\section{Concluding remarks}

Liu et al. (5) used the silicasomes to deliver irinotecan into PDAC tumors, observing an increase in survival from $29 \%$ without iRGD to $57 \%$ with iRGD. Finally, these authors correlated the iRGD effect with availability of NRP-1 in the tumor by using antibody inhibition and by comparing tumors with high and low NRP-1 expression. These results and those of Sugahara et al. (8) and Akashi et al. (14) emphasize the value of NRP-1 as a potential marker for patient stratification in a clinical trial projected to start in about a year.

\section{Acknowledgments}

The author's work is supported by grants R01CA188883 (to ER), R44 CA183287 to EnduRx Pharmaceuticals, and Cancer Center Support Grant CA30199 to Sanford Burnham Prebys from the National Cancer Institute.

Address correspondence to: Erkki Ruoslahti, Cancer Research Center, Sanford Burnham Prebys Medical Discovery Institute, 10901 N. Torrey Pines Rd., La Jolla, California 92037, USA. Phone: 858.646.3100, ext. 3101; E-mail: ruoslahti@sbpdiscovery.org.

1. Olive KP, et al. Inhibition of Hedgehog signaling enhances delivery of chemotherapy in a mouse model of pancreatic cancer. Science. 2009;324(5933):1457-1461.

2. Jain RK, Baxter LT. Mechanisms of heterogeneous distribution of monoclonal antibodies and other macromolecules in tumors: significance of elevated interstitial pressure. Cancer Res. 1988;48(24 pt 1):7022-7032.

3. Heldin CH, Rubin K, Pietras K, Ostman 
A. High interstitial fluid pressure - an obstacle in cancer therapy. Nat Rev Cancer. 2004;4(10):806-813.

4. Cho K, Wang X, Nie S, Chen ZG, Shin DM. Therapeutic nanoparticles for drug delivery in cancer. Clin Cancer Res. 2008;14(5):1310-1316

5. Liu X, et al. Tumor-penetrating peptide enhances transcytosis of silicasome-based chemotherapy for pancreatic cancer. JClin Invest. 2017;127(5):2007-2018.

6. Meng $\mathrm{H}$, et al. Use of a lipid-coated mesoporous silica nanoparticle platform for synergistic gemcitabine and paclitaxel delivery to human pancreatic cancer in mice. ACS Nano. 2015;9(4):3540-3557.

7. Liu X, et al. Irinotecan delivery by lipid-coated mesoporous silica nanoparticles shows improved efficacy and safety over liposomes for pancreatic cancer. ACS Nano. 2016;10(2):2702-2715.

8. Sugahara KN, et al. Tissue-penetrating delivery of compounds and nanoparticles into tumors. Cancer Cell. 2009;16(6):510-520.

9. Sugahara KN, et al. Coadministration of a tumor-penetrating peptide enhances the efficacy of cancer drugs. Science. 2010;328(5981):1031-1035.

10. Pierschbacher MD, Ruoslahti E. Cell attachment activity of fibronectin can be duplicated by small synthetic fragments of the molecule. Nature. 1984;309(5963):30-33.

11. Teesalu T, Sugahara KN, Kotamraju VR, Ruoslahti E. C-end rule peptides mediate neuropilin-1-dependent cell, vascular, and tissue penetration. Proc Natl Acad Sci U S A. 2009;106(38):16157-16162.

12. Pang HB, et al. An endocytosis pathway initiated through neuropilin-1 and regulated by nutrient availability. Nat Commun. 2014;5:4904.

13. Agemy L, et al. Targeted nanoparticle enhanced proapoptotic peptide as potential therapy for glioblastoma. Proc Natl Acad Sci U S A. 2011;108(42):17450-17455.

14. Akashi Y, et al. Anticancer effects of gemcitabine are enhanced by co-administered iRGD peptide in murine pancreatic cancer models that overexpressed neuropilin-1. Br JCancer. 2014;110(6):1481-1487.

15. Schmithals $C$, et al. Improving drug penetrability with $i R G D$ leverages the therapeutic response to Sorafenib and Doxorubicin in hepatocellular carcinoma. Cancer Res. 2015;75(15):3147-3154.

16. Sha $\mathrm{H}$, et al. Tumor-penetrating peptide fused EGFR single-domain antibody enhances cancer drug penetration into 3D multicellular spheroids and facilitates effective gastric cancer therapy. JControl Release. 2015;200:188-200.

17. Ruoslahti E. Tumor penetrating peptides for improved drug delivery [published online ahead of print April 1, 2016]. Adv Drug Deliv Rev. https://doi.org/10.1016/j.addr.2016.03.008.

18. Rudnick SI, et al. Influence of affinity and antigen internalization on the uptake and penetration of Anti-HER2 antibodies in solid tumors. Cancer Res. 2011;71(6):2250-2259.

19. Park JH, Gu L, von Maltzahn G, Ruoslahti E, Bhatia SN, Sailor MJ. Biodegradable luminescent porous silicon nanoparticles for in vivo applications. Nat Mater. 2009;8(4):331-336.

20. Mantis C, Kandela I, Aird F, Reproducibility Project: Cancer Biology. Replication Study: coadministration of a tumor-penetrating peptide enhances the efficacy of cancer drugs. eLife. 2017;6:e17584. 